\title{
Video Article \\ Visualizing the Effects of a Positive Early Experience, Tactile Stimulation, on Dendritic Morphology and Synaptic Connectivity with Golgi-Cox Staining
}

\author{
Richelle Mychasiuk ${ }^{1}$, Robbin Gibb ${ }^{1}$, Bryan Kolb ${ }^{1}$ \\ ${ }^{1}$ Canadian Centre for Behavioural Neuroscience, University of Lethbridge \\ Correspondence to: Richelle Mychasiuk at r.mychasiuk@uleth.ca \\ URL: https://www.jove.com/video/50694 \\ DOI: doi:10.3791/50694
}

Keywords: Neuroscience, Issue 79, Brain, Prefrontal Cortex, Neurons, Massage, Staining and Labeling, mPFC, spine density, methodology, enrichment

\section{Date Published: 9/25/2013}

Citation: Mychasiuk, R., Gibb, R., Kolb, B. Visualizing the Effects of a Positive Early Experience, Tactile Stimulation, on Dendritic Morphology and Synaptic Connectivity with Golgi-Cox Staining. J. Vis. Exp. (79), e50694, doi:10.3791/50694 (2013).

\section{Abstract}

To generate longer-term changes in behavior, experiences must be producing stable changes in neuronal morphology and synaptic connectivity. Tactile stimulation is a positive early experience that mimics maternal licking and grooming in the rat. Exposing rat pups to this positive experience can be completed easily and cost-effectively by using highly accessible materials such as a household duster. Using a cross-litter design, pups are either stroked or left undisturbed, for $15 \mathrm{~min}$, three times per day throughout the perinatal period. To measure the neuroplastic changes related to this positive early experience, Golgi-Cox staining of brain tissue is utilized. Owing to the fact that Golgi-Cox impregnation stains a discrete number of neurons rather than all of the cells, staining of the rodent brain with Golgi-Cox solution permits the visualization of entire neuronal elements, including the cell body, dendrites, axons, and dendritic spines. The staining procedure is carried out over several days and requires that the researcher pay close attention to detail. However, once staining is completed, the entire brain has been impregnated and can be preserved indefinitely for ongoing analysis. Therefore, Golgi-Cox staining is a valuable resource for studying experience-dependent plasticity.

\section{Video Link}

The video component of this article can be found at https://www.jove.com/video/50694/

\section{Introduction}

Although there are many reported and utilized techniques for the examination of negative early experiences on brain maturation, such as perinatal stress ${ }^{1,2}$, sensory deprivation ${ }^{3}$, and drug toxicity ${ }^{4}$, there are very few methodologies employed to examine the effects of positive experiences in this time period. Aside from environmental enrichment, tactile stimulation is one of a few brain enhancing treatments with demonstrated effects ${ }^{5}$. Tactile stimulation is a method of sensory stimulation to the skin that mimics the maternal rat behavior, licking and grooming. Its general acceptance as a positive manipulation arises from studies indicating that tactile stimulation improved maturation of premature infants and newborn rats ${ }^{6}$. In addition, research in the Meaney laboratory ${ }^{7}$ has demonstrated that higher levels of maternal licking and grooming are linked to positive outcomes in offspring. Owing to these positive influences, tactile stimulation rapidly evolved into a remedial strategy aimed at reducing anxiety ${ }^{8}$, improving outcomes associated with brain injury ${ }^{9-11}$, and attenuating drug sensitization ${ }^{12}$. As such, tactile stimulation is a valuable technique for the promotion of positive early experiences, with a proven ability to dramatically reorganize neuronal morphology and synaptic connectivity of the developing brain.

In order to examine and quantify changes in neuronal morphology, it is necessary to visualize intact neurons. The Golgi-Cox staining procedure is a modification of Camillo Golgi's technique published in the late 1800 s that provides discrete staining of a small number of complete neurons

${ }^{13}$. Although the procedure appears to stain neurons at random and reproducibility is commonly cited as major flaw, the small impregnation rate permits visualization of the entire neuronal element, including cell bodies, dendrites, dendritic spines, and axons. Similarly, the time required to impregnate a given brain with Golgi solution has also been cited as a downfall. However, given that once staining is complete tissue can be preserved indefinitely, and the time between perfusion of the brain and visualization of neurons with a microscope can be completed in less than 21 days, the time period is not unreasonable. In addition, with minor modifications to the protocol, Golgi-Cox staining can be effectively used to impregnate brains of rodents from all age ranges. As changes at the structural level have been linked to persistent modifications to behavioral and psychological functioning, the Golgi-Cox staining technique provides researchers with a valuable tool to measure neuroplasticity.

\section{Protocol}

All experiments were carried out in accordance with the Canadian Council of Animal Care and approved by the University of Lethbridge, Animal Care Committee. 


\section{Breeding and Tactile Stimulation}

1. Order pregnant rats from common animal suppliers or breed pups in-house using standard laboratory breeding procedures.

2. House all animals in a temperature controlled breeding room $\left(21^{\circ} \mathrm{C}\right)$, maintained on a $12: 12 \mathrm{hr}$ light:dark cycle and provide access to food and water ad libitum.

3. When pups are born, house female rats individually with their offspring

4. To avoid additional stress related to handling immediately after birth, commence tactile stimulation of pups at P3.

5. Tactile stimulation should be carried out three times per day (9:00 AM, 1:00 PM, and 4:00 PM) from P3-P21 and pups should be weaned from their mothers at P21.

6. When using a cross litter design, half of the pups from each litter will undergo tactile stimulation with the other half serving as controls. Randomly assign equal numbers of male and female pups to each group. To differentiate pups undergoing tactile stimulation and controls, mark one group of pups with a permanent marker on their hind legs and tail. Marking has to be re-applied every other day.

\section{Tactile Stimulation}

7. Remove dams from their home cage and place them in a temporary cage with food and water. Keep the dam and temporary cage in the breeding/housing room.

8. Weigh rat pups as a group prior to the morning tactile stimulation session (9:00 AM). For each session, transport rat pups their home cages to a separate testing room for tactile stimulation. Place the home cage on a heating pad that is set to $24{ }^{\circ} \mathrm{C}$.

9. Use a stiff board to divide the home cage into two halves. Place rat pups in the tactile stimulation group one half and control pups in the other half. Set a timer to $15 \mathrm{~min}$.

10. Using a soft feather-like duster, brush all pups in the tactile stimulation group at the same time for 15 consecutive min. The young rat pups ( P3-P12) ususally huddle together and appear to enter a deep sleep cycle making it very easy to stimulate all pups at once. As the pups age, they become more active, with some pups walking and investigating during the session. The experimenter should continuously move wandering pups into the group to ensure each pup receives equal stimulation.

11. Once the 15 min session is complete, transport the pups back to the breeding room and return the mother to the cage.

12. Repeat this process three times a day for the 19 days of tactile stimulation. After the final tactile stimulation session at P21, the pups should be weaned from their mothers. Pups should then be housed in cages with 5 or 6 other weaned animals of the same-sex.

13. If rats are undergoing no additional testing, they should be left undisturbed, on the normal light:dark cycle with access to food and water ad libitm (aside from regular cage cleaning and handling) until they reach approximately 100 days of age. If undergoing other experimental manipulations, animals should be treated or tested in accordance with those laboratory protocols.

\section{Sacrifice and Golgi-Cox Staining}

1. At approximately P100, administer an overdose of sodium pentobarbital via I.P. injection to the rats and perfuse intracardially with approximately $100 \mathrm{ml}$ of $0.9 \%$ saline.

2. Extract the brains when the perfusion is complete. Remove the brains from the skull, cutting the optic nerve underneath with effort to keep the cerebellum intact; researchers may choose to keep or remove the olfactory bulbs. Place extracted brains in an opaque Nalgene bottle with 20 $\mathrm{ml}$ of Golgi-Cox solution ${ }^{14}$.

3. Keep the brains in Golgi-Cox solution for 14 days. After 14 days, replace the Golgi-Cox solution with a $30 \%$ sucrose solution. Keep the brains in the sucrose solution for $2-5$ days before sectioning.

Note: If brains cannot be sectioned within 14 days of transfer to the sucrose solution, the researcher needs to replace the sucrose with a new stock of $30 \%$ sucrose. The researcher may replace the sucrose every two weeks for 4 months with no adverse effects on staining.

4. In order to section the brain, the brain should be blotted dry and fixed to the sectioning stage with cyanocacrylic glue. To prevent tearing or uneven sectioning, the researcher must be cautious and ensure that the entire brain is firmly secured to the stage.

5. The vibratome reservoir must be filled with $6 \%$ sucrose solution to a level that covers the sectioning blade. Set the vibratome parameters to a speed and amplitude to 5 , (the midpoint on both scales). Slice the brain into $200 \mu \mathrm{m}$ sections and place the sections on a $2 \%$ gelatinized microscope slide. Be sure to keep the sections wet during the course of sectioning.

6. When all of the sections of interest have been collected, press the sections onto the slides by applying pressure to the slides with moistened bibulous paper. Store the slides in a slide rack in the humidity chamber until they are ready to be stained. They should remain in the humidity chamber for at least $12 \mathrm{hr}$, but not longer than 4 days. The slides need to be moist and should not be permitted to dry out.

7. Prepare the staining regiment before commencing the staining process. Label twelve glass staining dishes $(10.7 \times 8.5 \times 6.8 \mathrm{~cm})$ and process in the following manner:

1. Distilled Water - 1 min

2. Ammonium Hydroxide -30 min (in the dark)

3. Distilled Water -1 min

4. Kodak Fix for Film - 30 min (in the dark)

5. Distilled Water $-1 \mathrm{~min}$

6. $50 \%$ Alcohol $-1 \mathrm{~min}$

7. $70 \%$ Alcohol - $1 \mathrm{~min}$

8. $95 \%$ Alcohol -1 min

9. $100 \%$ Alcohol $-5 \mathrm{~min}$

10. $100 \%$ Alcohol $-5 \mathrm{~min}$

11. Solution (1/3 Chloroform, $1 / 3$ HemoDe, $1 / 3100 \%$ Alcohol) - 15 min

12. HemoDe - 15 min 
* Xylene can be used in replace of HemoDe. In order to ensure consistent staining quality, fresh solutions should be used for each slide rack processed.

8. Following the last 15 min emersion in HemoDe, coverslip the slides with Permount.

9. Allow the slides to air dry before examining with a microscope.

\section{Representative Results}

When this staining procedure has been followed appropriately, consistent and uniform staining of dendrites and spines is generated. See Figure 1 for a representation of Golgi-Cox neuronal staining. This procedure produces staining that is comparable to the newly developed in vitro methods and may permit visualization of dendritic fields that are uninterruptable following tissue embedding. The vibratome based technique for Golgi-Cox staining has been found to produce more widespread staining of terminal branches and pyramidal neurons, than staining with either celloidin-embedded and cryostat-sectioned tissue, ${ }^{15}$. Furthermore, because this method of staining impregnates the entire brain, all sections can be analyzed either immediately or in the future. As the location of morphological change is it is not always obvious when generating an original hypothesis, this process allows for the exploration of additional brain regions and prevents disposal of tissue that may be useful in the future.

This staining method can be used for reliable staining of any aged rat brains (P0-old age). However, when staining young brains (<1 gram), the brain should only remain in the Golgi-Cox solution for 6 days, rather than the 14 days recommended for adult brains; all other procedures are held constant. The main difficulty that may be encountered during this Golgi-Cox staining process is the inability to produce high-quality staining of central brain regions, including the thalamus. As the entire brain is impregnated at the same time, central regions may not receive ideal concentrations of stain. Despite this finding, the Golgi-Cox technique produces exceptional staining of subcortical regions such as the hippocampus and striatum. A final limitation of the procedure arises from incomplete removal of blood from the brain during the perfusion process. Blood may produce artifacts in the brain tissue that makes it difficult to photograph and trace the neurons.

Golgi-Cox staining techniques provide a reliable measure of dendritic morphology and neuroanatomy that is useful for an extensive range of studies designed to examine changes at the neuronal level. When examining neuronal structure in the prefrontal cortex following tactile stimulation in the early-postnatal period, Golgi-Cox staining revealed dramatic increases in dendritic complexity. Anatomical changes related to early tactile stimulation can be demonstrated visually by comparing spine density of pyramidal neurons from a control rat to pyramidal neurons from a rat that underwent tactile stimulation (see Figure 1). In addition, parameters such as dendritic arborization, dendritic length, and spine density can be calculated and statistically analyzed to generate continuous data that can be compared across animals. To generate reliable results, analysis should be carried out on a minimum of three animals per treatment group and approximately five neurons per hemisphere should be randomly selected for each brain region under analysis. Figure $\mathbf{2}$ demonstrates the dendritic complexity (spines/ $\mu \mathrm{m} \times \mu \mathrm{m}$ of dendrite) of animals that received tactile stimulation and animals that did not. In both the apical and basilar fields of the neurons analyzed from the mPFC (Cg3), tactile stimulation resulted in proliferation of neuronal parameters. 


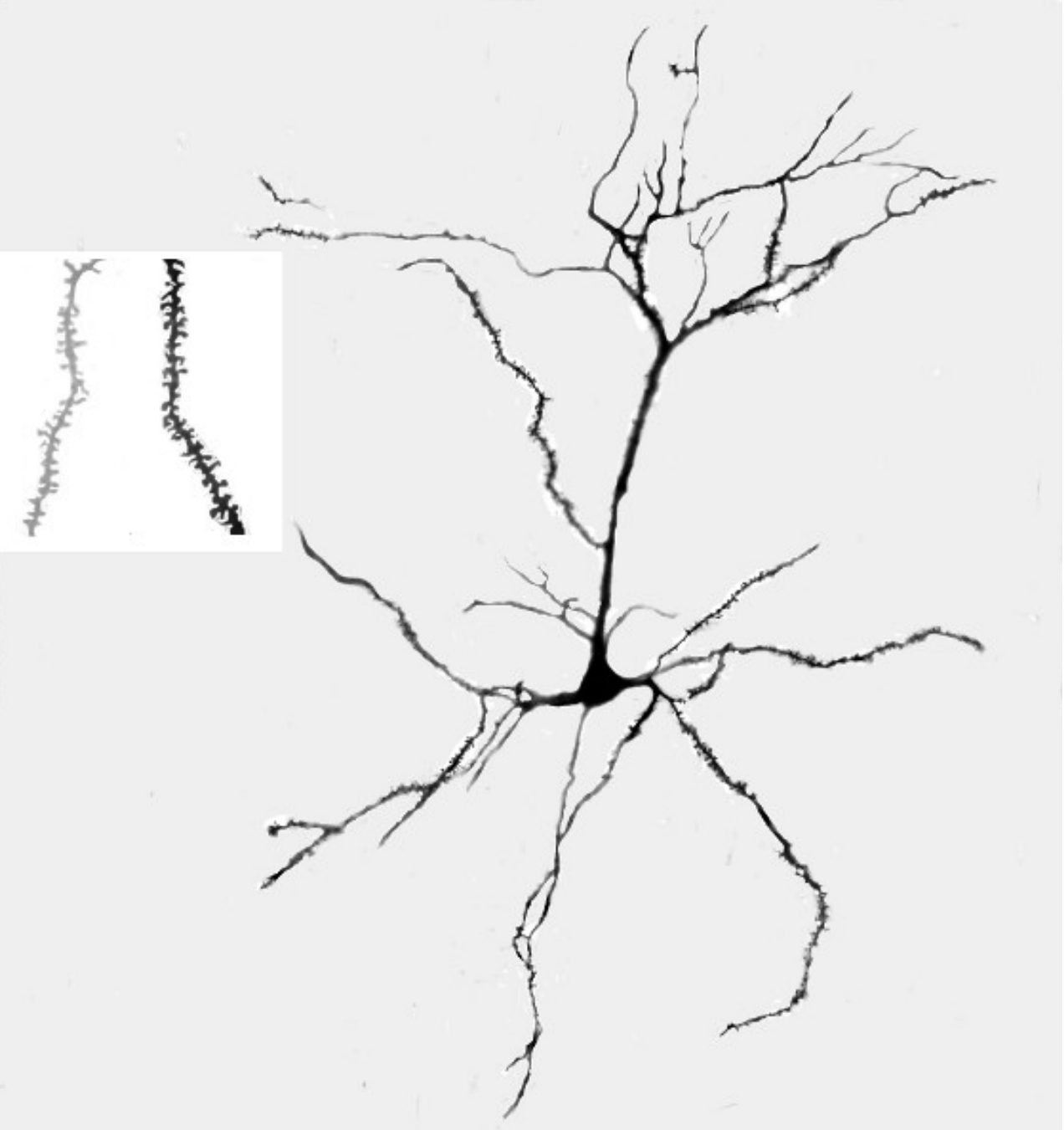

Figure 1. A. Representative photograph of a Golgi-Cox stained pyramidal neuron from layer III of area Cg3 in a rat that received tactile stimulation during development. B. Representative magnified $(1,000 \mathrm{X})$ photograph of dendritic spines on the terminal dendrites in the basilar field of Cg3; the lighter image on the left is for a rat in the control group and the darker image on the right is from a rat who received tactile stimulation.

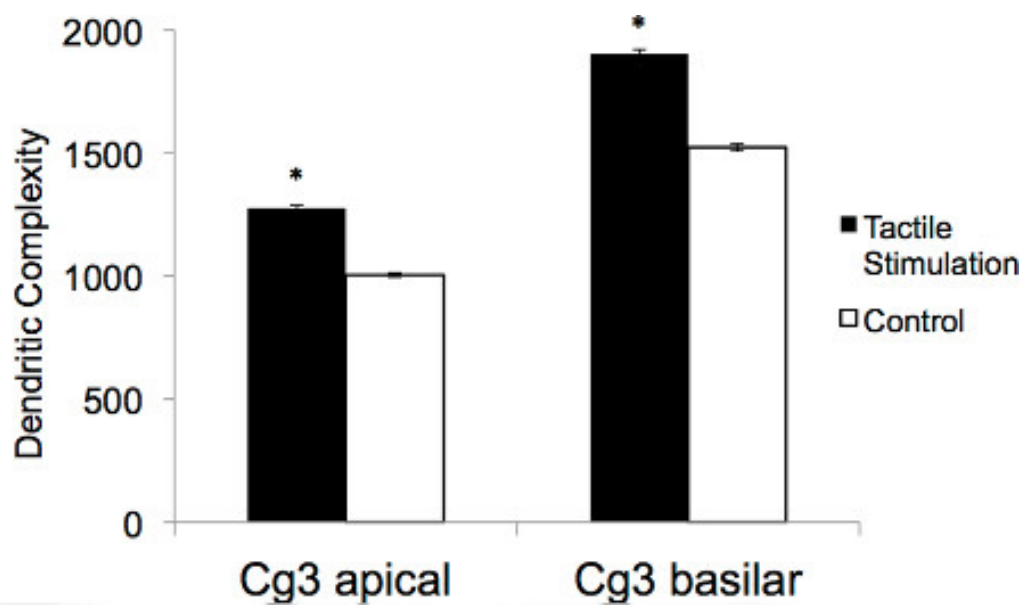

Figure 2. Illustrative representation of the average apical and basilar dendritic complexity (spines/ $\mu \mathrm{m} \times \mu \mathrm{m}$ ) for neurons in the $\mathrm{Cg} 3$ area from adult rats that either received tactile stimulation during development or did not $\left({ }^{*} p<0.01\right)$. 


\section{Discussion}

Owing to the overt plasticity of the developing brain, it is important that experimental procedures involving early experiences are thoroughly reviewed and attempts are made to control for all intervening variables. For this reason, a cross litter design is employed during the tactile stimulation procedure to ensure that pups are receiving similar experiences in all other domains of development. In addition, it is also important that multiple pups from multiple litters are selected for analysis to avoid the possibility that effects result from a bias in a single pup or litter.

Tactile stimulation is believed to mimic the naturally occurring maternal behavior, licking and grooming, which is thought to be beneficial to offspring development. Although prior research has emphasized the first week of life (P0-P7) to be a critical period for licking and grooming ${ }^{16}$ the sheer magnitude of change identified following 18 days of tactile stimulation may imply that although sensitive times exist, longer exposure is superior. It is also important to note that pups receiving tactile stimulation in this experimental paradigm also experience licking and grooming by their mothers, hence the tactile stimulation is in addition to normal stimulation administered by the mother. Finally, one must also be cognizant of region-dependent changes. Although tactile stimulation during development increased dendritic complexity in the prefrontal cortex, this does not guarantee that structural changes of this nature will be evident in all brain regions. It is possible that neuronal morphology in other brain regions such as the parietal cortex, would respond in a strikingly different manner to the same experience. However, because the tactile stimulation procedure is easily administered and poses no risk to the offspring or dams, it has the potential to serve as a valuable tool for many research studies aimed at improving developmental outcomes.

With respect to Golgi-Cox staining of brain tissue, the critical steps for successful visualization of neuronal cells are as follows: 1) there must be adequate perfusion of the brain with saline solution. Improper or insufficient perfusion of the brain tissue results in blood vessel artifacts that make it difficult to actually visualize neuronal cells through the maze of blood vessels, while also complicating the ability to photograph stained neurons. 2) Perfused brains should be stored in Golgi-Cox solution and sucrose solution in the dark. Storing the brain tissue in the dark reduces the background staining of the tissue, again increasing the chance of successfully visualizing quality neuronal cells. 3) Brain tissue is stored in sucrose solution following the 14 day storage in Golgi-Cox solution. When the tissue is immersed in the $30 \%$ sucrose solution for $2-5$ days, brains are more pliable which prevents shattering and tearing of sections when cutting. It is important to prevent brain tissue from remaining in the sucrose solution for increased time periods (unless the sucrose solution is continuously replaced with fresh solution) because prolonged storage in sucrose reduces the staining quality. 4) Finally, once slides have been stained and cover slipped, they should be given adequate time to dry before visualization with a microscope. If slides are not permitted to adequately air dry, the tissue may darken, reducing successful visualization of cortical neurons.

Stable changes in psychological functioning and behavioral responses that occur in response to experiences are believed to be facilitated by reorganization of neuronal morphology and synaptic connectivity ${ }^{17}$. As these structural changes provide a measurable resource for experiencedependent plasticity, the use of a reliable staining procedure is important. This vibratome based Golgi-Cox staining procedure provides reliable staining of fine branches and dendritic spines that may not be evident with other protocols such as celloidin-embedding. The distinctiveness of the Golgi-Cox procedure stems from its capacity to only stain a small fraction of neuronal elements (1-10\%), which permits tracing of single neurons for long distances. Despite the fact that only a small fraction of neurons are impregnated with the stain, cells that are rendered visible, maintain all features including cell body, dendrites, dendritic spines and axons. Moreover, when the staining procedure is carried out correctly, the stained cells stand out clearly and distinctly against a transparent background because other cortical structures remain unstained and transparent. Owing to the realization that persistent changes in outward functioning must be related to the plasticity of the nervous system, i.e. the capability of neurons to modify their structure and connectivity, the Golgi-Cox staining procedure provides researchers with a reliable technique to visualize and quantify this plasticity.

\section{Disclosures}

The authors declare that we have no competing financial interests.

\section{Acknowledgements}

This work is funded by NSERC grants to BK and RG. The authors would also like to thank Kehe Xie and Russell Hosain for their Golgi-Cox staining expertise.

\section{References}

1. Mychasiuk, R., Ilnystkyy, S., Kovalchuk, O., Kolb, B., \& Gibb, R. Intensity matters: Brain, behaviour, and the epigenome of prenatally stressed rats. Neuroscience. 180, 105-10 (2011).

2. Muhammad, A., Carroll, C., \& Kolb, B. Stress during development alters dendritic morphology in the nucleus accumbens and prefrontal cortex. Neuroscience. 216, 103-9 (2012).

3. Wiesel, T. \& Hubel, D. Effects of visual deprivation on morphology and physiology of cells in the cat's lateral geniculate body. Journal of Neurophysiology. 26(978), 6 (1963).

4. Dwyer, J., McQuown, S., \& Leslie, F. The dynamic effects of nicotine on the developing brain Pharmacology \& Therapeutics. 122, 125-39 (2009).

5. Richards, S., Mychasiuk, R., Kolb, B., \& Gibb, R. Tactile stimulation during development alters behaviour and neuroanatomical organization of normal rats. Behavioural Brain Research. 231, 86-91 (2012).

6. Schanberg, S. \& Field, T. Sensory deprivation stress and supplemental stimulation in the rat pup and preterm neonate. Child Development. 58(6), 1431-47 (1987). 
7. Caldji, C., Tannenbaum, J., Sharma, S., Francis, D., Plotsky, P., \& Meaney, M. Maternal care during infancy regulates the development of neural systems mediating the expression of fearfulness in the rat. Proceedings of the National Academy of Sciences. 95(9), 5335-40 (1998).

8. Imanaka, A., Morinobu, S., Toki, S., Yamamoto, S., Matsuki, A., Kozuru, T., et al. Neonatal tactile stimulation reverses the effect of neonatal isolation on open-field and anxiety-like behavior, and pain sensitivity in male and female adult Sprague-Dawley rats. Behavioural Brain Research. 186, 91-7 (2008).

9. Gibb, R., Gonzalez, C., Wegenast, W., \& Kolb, B. Tactile stimulation promotes motor recovery following cortical injury in adult rats. Behavioural Brain Research. 214(1), 102-7 (2010).

10. Rodrigues, A., Artneni, N., Abel, C., Zylbersztejn, D., Chazan, R., Viola, G., et al. Tactile stimulation and maternal separation prevent hippocampal damage in rats submitted to neonatal hypoxia-ischemia. Brain Research. 1002, 94-9 (2004).

11. Kolb, B. \& Gibb, R. Tactile stimulation after frontal or parietal cortical injury in infant rats facilitates functional recovery and produces synaptic changes in adjacent cortex. Behavioural Brain Research. 214:115-20 (2010).

12. Muhammad, A., Hossain, S., Pellis, S., \& Kolb, B. Tactile stimulation during development attenuates amphetamine sensitization and structurally reorganizes prefrontal cortex and striatum in a sex-dependent manner. Behavioral Neuroscience. 125(2), 161-74 (2011).

13. Golgi, C. Sulla struttura della sostanza grigia dell cervello. Gaz Med Lomb. 33, 244-6 (1873).

14. Glaser, E.M. \& van der Loos, H. Analysis of thick brain sections by obverse-reverse computer microscopy: Application of a new, high clarity Golgi-Nissl stain. Journal of Neuroscience Methods. 4, 117-25 (1981).

15. Gibb, R. \& Kolb, B. A method for vibratome sectioning of Golgi-Cox stained whole rat brain. Journal of Neuroscience Methods. 79, 1-4 (1998).

16. Meaney, M. Maternal care, gene expression, and the transmission of individual differences in stress reactivity across generations. Annual Review of Neuroscience. 24, 1161-92 (2001).

17. Robinson, T.E. \& Kolb, B. Structural plasticity associated with exposure to drugs of abuse. Neuropharmacology. 47(Suppl 1), 33-46 (2004). 